Check for updates

Cite this: RSC Adv., 2017, 7, 55741

Received 7th September 2017

Accepted 11th November 2017

DOI: 10.1039/c7ra09992d

rsc.li/rsc-advances

\title{
Synthesis of comb-like polymeric surfactants with
a tricyclic rigid core and their use as dispersants in \\ Synthesis of comb-like polymeric surfactants with
a tricyclic rigid core and their use as dispersants in pymetrozine water suspension concentrates $\dagger$
}

\author{
Xinyan Yan, ${ }^{a}$ Zhaolan Zhai, ${ }^{a}$ Zhanqian Song, ${ }^{a}$ Shibin Shang (D) ab \\ and Xiaoping Rao (D)*ab
}

\begin{abstract}
Comb-like polymeric surfactants with a tricyclic rigid core have been synthesized from rosin. Their structures were confirmed by IR and ${ }^{1} \mathrm{H}$ NMR spectroscopies and GPC. The surface activities of these surfactants were evaluated according to critical micelle concentration (CMC), the surface tension at CMC ( $\left.\gamma_{C M C}\right)$, emulsification properties (EP), and foam properties (FP). Their CMC values were $0.004-$ $0.023 \mathrm{~g} \mathrm{~L}^{-1}$, which were much smaller than those of the surfactant without a rigid core $\left(14.59 \mathrm{~g} \mathrm{~L}^{-1}\right)$. Measurement of the rheological properties revealed that the surfactant solutions at $50 \mathrm{~g} \mathrm{~L}^{-1}$ were dilatant fluids. A break point in the viscosity-shear rate curve moved to a high shear rate when the proportion of the monomer with a rigid core increased. The dispersing and stabilizing properties of these surfactants as dispersants were investigated to prepare pymetrozine water suspension concentrates. The results indicated that surfactants with a rigid core had good grinding and dispersion effects, the suspension rate of the pymetrozine water suspension concentrates was higher than $95 \%$, and the water suspension concentrates had good stability during the cold storage process. The introduction of a rigid core into polymeric surfactants can effectively improve the surface activities of these surfactants and the suspension rate and stability of water suspension concentrates.
\end{abstract}

\section{Introduction}

With the increasing concern for the need to protect environment, it is urgent to develop green pesticide formulations. There are some formulations, including micro emulsion (ME), emulsion in water (EW), novel emulsifiable concentrate (EC), suspension concentrate (SC), micro capsule (MS), and so on, that can meet the requirements of the green pesticide formulation. ${ }^{1}$ A green pesticide formulation requires not only green raw pesticide but also green additives. Water or other green substances can be used to replace organic solvents such as methylbenzene and dimethylbenzene. ${ }^{2}$ Pymetrozine has a high application value in controlling aphids in crops; however, pymetrozine is a powder pesticide, and it will disperse in the air during processing and use, which is harmful to humans and animals. Water suspension concentrates can solve the above mentioned problems. Moreover, as compared to emulsifiable concentrates, water suspension concentrates do not require

${ }^{a}$ Institute of Chemical Industry of Forest Products, CAF, National Engineering Lab. for Biomass Chemical Utilization, Key and Open Lab. of Forest Chemical Engineering, SFA, Key Lab. of Biomass Energy and Material, Nanjing 210042, Jiangsu Province, China. E-mail: rxping2001@163.com

${ }^{b}$ Institute of Forestry New Technology, CAF, Beijing 100091, China

$\dagger$ Electronic supplementary information (ESI) available. See DOI: 10.1039/c7ra09992d a large number of organic solvents. Therefore, water suspension concentrates have many advantages. Alkylphenol ethoxylates (APEOs) have been often used as an emulsifier in the pesticide field due to their excellent emulsification and dispersion; however, APEO is toxic. ${ }^{3}$

Compared to conventional surfactants, polymeric surfactants have much higher structural complexity both in number and distribution of hydrophilic and hydrophobic moieties along the chain; this can result in very different behaviors. Therefore, they have drawn extensive attention in the last few decades, especially, in several fields such as in enhanced oil recovery, pharmacology, medicine, coatings, optoelectronics, nanotechnology, (mini) emulsion polymerizations, water purification, cosmetics biotechnology, electronics, and so on. ${ }^{4}$

Comb-like polymeric surfactant is a kind of special structure of conventional block copolymers. ${ }^{5}$ Comb-like poly(ethylene glycol) monomethyl ether (MPEG) graft polymeric surfactants can be synthesized by the MPEG reaction with a polymeric intermediate or by copolymerization of MPEG macromonomers with vinyl monomers. ${ }^{6}$

The raw materials required to prepare a surfactant are classified as natural or synthesized from petroleum, according to their origin. Increasing interest in natural, renewable, and biodegradable materials make natural polymers, such as cellulose and its derivatives, attractive raw materials to prepare biopolymeric surfactants. These surfactants have attracted 
significant attention because they easily biodegrade. In the preparation of biopolymeric surfactants, it is expected that the current trend of replacing petroleum raw materials with natural and sustainable raw materials will continue. ${ }^{7}$

Rosin is composed of a series of tricyclic diterpene resin acids. It is separated from oleoresin secreted from pine trees naturally, and it is regarded as biocompatible, biodegradable, and eco-friendly. ${ }^{\mathbf{8} 9}$ Like fatty acids, rosin has a carboxylic acid group in its skeleton and a tricyclic rigid core hydrophobic skeleton, which is more rigid than alkylphenol.

In the present study, comb-like polymeric surfactants were synthesized from the sustainable forest resource rosin. Their surface activities were investigated, and pymetrozine water suspension concentrates were prepared by the ball mill method using these surfactants as dispersants.

\section{Materials and methods}

\section{Materials}

Rosin was provided by the Richeng Forest Product Chemical Co. LTD (Guangxi province, China). Glycidyl methacrylate (GMA) was purchased from Aldrich. Methyl acrylate polyethylene glycol ester (MAPEG), with an average molecular weight of $950 \mathrm{Da}$, was purchased from Macklin. 2,2'-Azobisisobutyronitrile (AIBN, 99\%), absolute ethyl alcohol, triethylamine, ethylene glycol, sodium dodecyl sulfate, hydroquinone, and dialysis membrane (cut-off $3500 \mathrm{Da}$ ) were obtained from Sinopharm Chemical Reagent Co. LTD (China). Pymetrozine was obtained from Hengrong Business Companies (Jiangsu province, China). They were all used as received.

\section{Synthesis methods}

Rosin glycidyl methacrylate (RGMA) was prepared by epoxy ringopening esterification of rosin with GMA according to Scheme 1. Rosin and GMA (the molar ratio was $1: 1$ ) were put in a threenecked flask equipped with a reflux condenser, a magnetic stirrer, and a thermometer. Triethylamine (0.5 wt $\%)$ was designated as the catalyst, and hydroquinone (0.2 wt $\%)$ was designated as the inhibitor. Absolute ethyl alcohol was used as the solvent. The mixture was stirred at $80{ }^{\circ} \mathrm{C}$ for $7 \mathrm{~h}$. The structure of RGMA was confirmed by IR spectroscopy.

Poly(rosin glycidyl methacrylate ester-co-methylacrylic acid polyethylene glycol ester), (poly(RGMA-co-MAPEG)), was prepared by free radical polymerization of RGMA with<smiles>CC(C)C1=CC2=CCC3[C@](C)(C(=O)O)CCC[C@]3(C)C2CC1</smiles><smiles>C=C(C)C(=O)OCC1CO1</smiles>

GMA

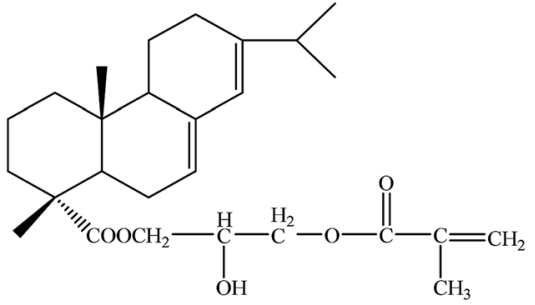

RGMA

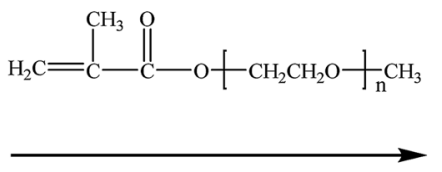

MAPEG

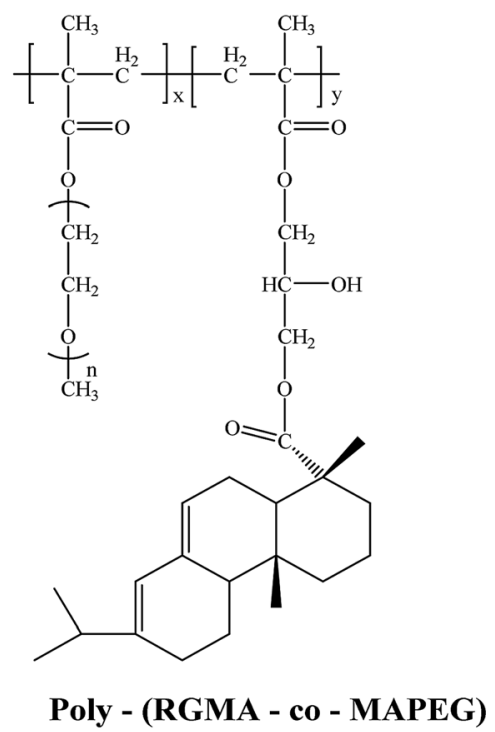


MAPEG950. RGMA and MAPEG950 were dissolved in absolute ethyl alcohol in a three-necked flask equipped with a reflux condenser, a thermometer, and a magnetic stirrer. The mixture was stirred at $60{ }^{\circ} \mathrm{C}$ for $1 \mathrm{~h}$. Then, the initiator AIBN (1 wt\%) was dissolved in a solvent and added dropwise. After all the initiator was added, the mixture was stirred at $80^{\circ} \mathrm{C}$ for $12 \mathrm{~h}$. Then, the solvent was removed by a rotary vacuum dryer. The crude products were purified by dissolving them in tetrahydrofuran and adding a large quantity of petroleum ether. The precipitated products were further purified by re-precipitation two times. The products were dried by vacuum drying. The products were dissolved in deionized water and dialyzed via a dialysis membrane $(3500 \mathrm{Da})$ for half a month to remove the unreacted monomers. The pure products poly(RGMA-co-MAPEG) were obtained after removing water using a freeze dryer. The structures of comb-like surfactants were characterized by IR and ${ }^{1} \mathrm{H}$ NMR spectroscopy and GPC.

\section{Characterization and performance}

\section{FTIR}

Fourier transform infrared (FT-IR) spectra were used to compare the structure of rosin, RGMA, and poly(RGMA-coMAPEG). The spectra were obtained using a Thermo Scientific Nicolet IS10 IR spectrophotometer by the ATR method over the range of $4000-500 \mathrm{~cm}^{-1}$ at $4 \mathrm{~cm}^{-1}$ resolution and averaged over 16 scans per sample.

\section{H-nuclear magnetic resonance $\left({ }^{1} \mathrm{H}\right.$ NMR)}

${ }^{1} \mathrm{H}$ NMR measurement was carried out using a Bruker Avance III 400 spectrometer operating at $400 \mathrm{MHz}$. Deuterated chloroform $\left(\mathrm{CDCl}_{3}\right)$ was used as the solvent.

\section{Gel permeation chromatography (GPC)}

Relative values of number-average $\left(M_{\mathrm{n}}\right)$ molecular weight, weight-average $\left(M_{\mathrm{w}}\right)$ molecular weight, and molecular weight distribution curves of poly(RGMA-co-MAPEG) were determined by GPC. GPC was performed at room temperature using a Malvern Viscotek 3580 System equipped with a Waters 2414 detector. The columns were Styragel HR1 and HR2 $(300 \mathrm{~mm} \times$ $7.8 \mathrm{~mm}$, two columns in series connection). The eluent was HPLC grade THF, and the flow rate was $1 \mathrm{~mL} \mathrm{~min}^{-1}$. The standard to generate the calibration curve was monodispersed polystyrene. All data were obtained using the Waters Breeze GPC software Version 5.3.1.4.

\section{Critical micelle concentration (CMC) and minimum surface tension $\left(\gamma_{\mathrm{CMC}}\right)$}

The surface tensions of these comb-like polymeric surfactants were measured at room temperature by a Wilhelmy plate T107 using a Sigma701 Automatic Surface Tensiometer. The width, thickness, height, and circumference of the Wilhelmy plate were $19.44 \mathrm{~mm}, 0.1 \mathrm{~mm}, 65 \mathrm{~mm}$, and $39.08 \mathrm{~mm}$, respectively. The software automatically tested the surface tension as a function of the mass concentration. The concentrations of surfactants in water were gradually increased from 0 to $0.5 \mathrm{~g} \mathrm{~L}^{-1}$ (the concentration of P-MAPEG was increased from $0 \mathrm{~g} \mathrm{~L}^{-1}$ to $\left.15 \mathrm{~g} \mathrm{~L}^{-1}\right) .{ }^{10}$ Their surface tension in water decreased markedly at low concentrations. When the concentration was higher, the surface tension reduced slightly. The concentration at the inflection point was the $\mathrm{CMC}$, and the surface tension at the inflection point was $\gamma_{\mathrm{CMC}} \cdot{ }^{11}$ All data were obtained from the One Attention software.

\section{Emulsification properties (EP)}

The emulsification properties (EP) were tested as follows: $40 \mathrm{~mL}$ of a $0.1 \mathrm{wt} \%$ surfactant aqueous solution and $40 \mathrm{~mL}$ liquid paraffin were added to a $100 \mathrm{~mL}$ mixing cylinder with a stopper. The cylinder was inverted up and down fifty times, successively. Then, the time for separation of $10 \mathrm{~mL}$ of water was determined. The experiment was repeated three times with high repeatability.

\section{Foam properties (FP)}

Foam properties (FP) were tested as follows: $20 \mathrm{~mL}$ of a $0.5 \mathrm{wt} \%$ surfactant aqueous solution and $10 \mathrm{~mL}$ pure water were added to a $100 \mathrm{~mL}$ mixing cylinder with a stopper. The cylinder was inverted up and down twenty five times, successively. Then, the height of the foam was initially determined, and then, it was determined again after five minutes. ${ }^{9}$ Each test was repeated three times, and the average of the foam height was taken.

\section{HLB values}

HLB values were calculated using the following equation: HLB $=23.64 \lg V-10.16$. Herein, $0.2 \mathrm{~g}$ of surfactant was dissolved in a $20 \mathrm{~mL}$ mixture of dioxane and benzene at $90: 4 \mathrm{v} / \mathrm{v}$. It was titrated by distilled water to obvious turbidity at $25^{\circ} \mathrm{C}$. $\mathrm{V}$, which in the formula represents the volume of distilled water used for titration to the end point, was determined. ${ }^{12}$

\section{The rheological properties}

The rheological properties were measured by controlled stress HAAKE MARS II equipped with a measuring geometry named the PP60Ti cylinder sensor system; ${ }^{13}$ the cylinder diameter was $6 \mathrm{~cm}$. The concentration of the surfactant aqueous solutions was $50 \mathrm{~g} \mathrm{~L}^{-1}$. The steady state shear experiment was carried out at $30 \pm 2{ }^{\circ} \mathrm{C}, 45 \pm 2{ }^{\circ} \mathrm{C}, 60 \pm 2{ }^{\circ} \mathrm{C}$, and the shear rate ranged from 0.1 to $600 \mathrm{~s}^{-1}$ with a period of $20 \mathrm{~min}$.

\section{Pymetrozine water suspension concentrates}

The formulation of water suspension concentration was as follows: pymetrozine ( 5 parts), dispersant ( 6 parts), the wetting agent sodium dodecyl sulfate ( 6 parts), and anti-freezing agent glycol ethylene glycol ( 2 parts). Room was left for water ( 81 parts). The mixture has been grinded by a small-scale ball mill at $200 \mathrm{rpm}$ for 3 hours. The diameter of the zirconium bead was $0.1 \mathrm{~mm}$. The thermal storage stability, cold storage stability, and the suspension rate of water suspension concentrates were measured according to GB/T19136-2003, GB/T19137-2003, and GB/T14825-2006, respectively. 


\section{Results and discussions}

\section{Characterization of the products}

A series of poly(RGMA-co-MAPEG) molecules were obtained by controlling the feed ratios of $0: 1,1: 2,1: 1$, and $2: 1$ of $n$ (RGMA) : $n$ (MAPEG950). The average relative molecular mass of MAPEG was 950; thus, the samples were labeled as 950AA, 950BA, 950CA, and 950DA. The intermediate product RGMA and the comb-like polymeric surfactants poly(RGMA-coMAPEG) were prepared successfully according to Scheme 1 . The FTIR spectra of rosin, RGMA, and poly(RGMA-co-MAPEG) are shown in Fig. S1, $\dagger$ and the ${ }^{1} \mathrm{H}$ NMR spectra of poly(RGMA-coMAPEG) and poly-MAPEG are displayed in Fig. S2. $\uparrow$ The IR and ${ }^{1} \mathrm{H}$ NMR analyses results indicated that the intermediate and target products were successfully prepared.

Since the final products were purified and did not have unreacted monomer RGMA and MAPEG950, the ratio of hydrophobic monomer RGMA to hydrophilic monomer MAPEG950 $(x: y, R)$ in the poly(RGMA-co-MAPEG) could be calculated from the ${ }^{1} \mathrm{H}$ NMR peak ratio between the ethylene protons of RGMA $(\delta$ $5.75)$ and the methyl protons of MAPEG ( $\delta 3.34)$. Therefore, $x: y$ can be evaluated using the following equation:

$$
R=\frac{x}{y}=\frac{A_{\mathrm{s}} / 1}{A_{\mathrm{m}} / 3}
$$

where $A_{\mathrm{s}}$ is the peak areas of the ethylene protons and $A_{\mathrm{m}}$ is the methyl protons in the ${ }^{1} \mathrm{H}$ NMR spectrum. ${ }^{14}$ The results of calculations are shown in Table 1.

The GPC results of comb-like surfactants after re-precipitation and dialysis are summarized in Table 1 . The GPC curves obtained after purification are displayed in Fig. S3, $\uparrow$ and the GPC curves obtained before purification are displayed in Fig. S4. $\dagger$ The polydispersity index of our product was small because the crude products were purified by re-precipitation and dialysis to remove the unreacted monomers and some polymers with a lower molecular weight. The polydispersity index can be calculated $\left(M_{\mathrm{w}}\right)$ $M_{\mathrm{n}}$ ), which can measure the distribution of molecular weight. It could be concluded that the polydispersity index increased as $R$ increased. This may be caused by the special structure of rosin. The greater the quantity of the hydrophobic group RGMA in the comb-like polymeric surfactants, the greater the steric hindrance. Thus, the composition of the comb-like surfactants could be controlled by controlling the feed ratios of RGMA and MAPEG.

\section{Surface activities}

The CMC and $\gamma_{\mathrm{CMC}}$ curve of poly-MAPEG (950AA) and poly(RGMA-co-MAPEG) are shown in Fig. 1 and 2, respectively. At first, the surface tension of these surfactants in water decreased sharply at lower concentrations. Then, the surface tension slightly decreased at a higher concentration. Finally, the surface tension remained constant. ${ }^{15}$ The turn-off in the curve was the CMC of the surfactant. As shown in Fig. 2, the CMC value of 950AA was $14.59 \mathrm{~g}$ $\mathrm{L}^{-1}$ and $\gamma_{\mathrm{CMC}}$ was $35.1 \mathrm{mN} \mathrm{m}^{-1}$. As shown in Fig. 2 , the CMC value of 950BA, 950CA, and 950DA was 0.004, 0.011, and $0.023 \mathrm{~g} \mathrm{~L}^{-1}$, and $\gamma_{\mathrm{CMC}}$ was $57.8,56.6$, and $51.9 \mathrm{mN} \mathrm{m}^{-1}$, respectively. It can be concluded that the $\mathrm{CMC}$ values of poly(RGMA-co-MAPEG) are dramatically lower than the CMC values of poly-MAPEG. This may be because the hydrophobic property of the comb-like polymeric surfactants was improved. With an increase in the ratio of the hydrophobic group RGMA in the molecule, the trend of molecular adsorption on the surface was stronger. However, the CMC values of poly(RGMA-co-MAPEG) increased slightly with the increasing proportion of the hydrophobic rigid core in poly(RGMA-co-MAPEG). This may be caused by the steric hindrance of the tricyclic rigid core. However, the increase of the hydrophobic property was the main reason that the molecular micelle could form in solutions at lower concentrations.

HLB values, emulsification properties, and foam properties of these surfactants are displayed in Table 2 . HLB values can reflect the hydrophilic-lipophilic balance value of the surfactant. The higher the value of HLB, the stronger the hydrophilicity of the surfactant. Conversely, the lower the value of HLB, the stronger the lipophilicity of the surfactant. With the increment of $R$ value, the HLB value of poly(RGMA-co-MAPEG) decreased gradually, and the hydrophilicity of the surfactants was weakened. However, the

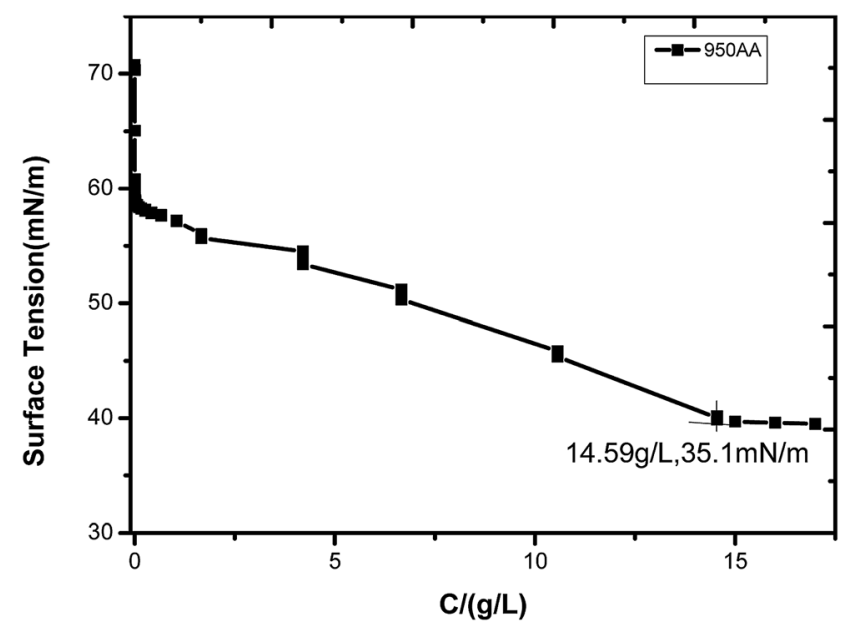

Fig. 1 CMC and $\gamma_{C M C}$ curve of poly-MAPEG.

Table 1 Effect of the feed ratio of $n$ (RGMA) : $n$ (MAMPEG) on the composition of poly(RGMA-CO-MAPEG)

\begin{tabular}{|c|c|c|c|c|c|}
\hline & $\begin{array}{l}\text { Feed ratio of } \\
n(\mathrm{RGMA}): n \text { (MAPEG) }\end{array}$ & $M_{\mathrm{n}}$ & $M_{\mathrm{w}}$ & $\begin{array}{l}\text { Polydispersity } \\
\text { index }\end{array}$ & $\begin{array}{l}\text { Composition of } \\
\text { surfactants }(R)\end{array}$ \\
\hline 950AA & $0: 1$ & 7940 & 8892 & 1.12 & 0 \\
\hline 950BA & $1: 2$ & 7969 & 9403 & 1.18 & 0.2 \\
\hline 950CA & $1: 1$ & 6336 & 8153 & 1.28 & 0.92 \\
\hline 950DA & $2: 1$ & 7271 & 9966 & 1.37 & 1.12 \\
\hline
\end{tabular}




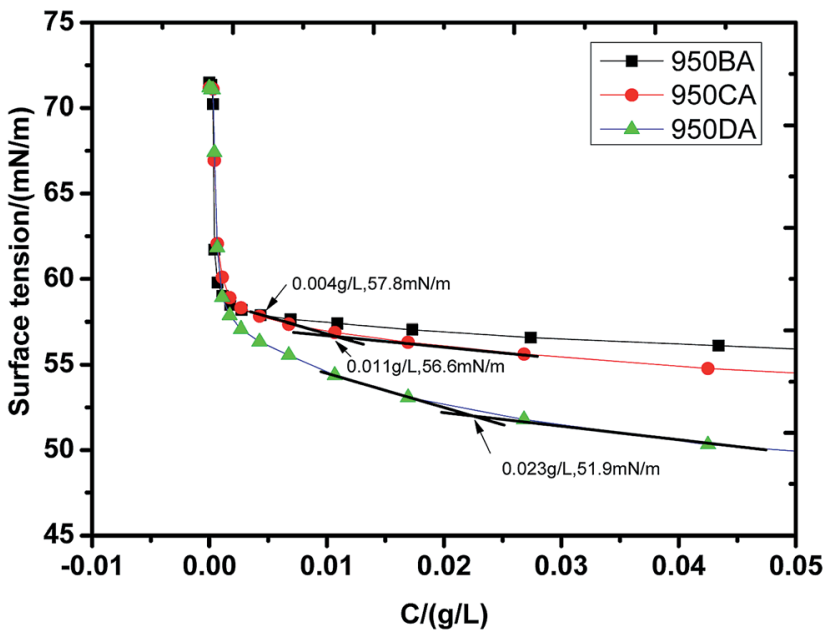

Fig. 2 CMC and $\gamma_{C M C}$ curve of poly(RGMA-CO-MAPEG).

HLB values of these surfactants were greater than 17 . Thus, all these surfactants are hydrophilic. They can be applied to the $\mathrm{O} / \mathrm{W}$ emulsifier, detergent, solubilizer, and so on. With the increment of the $R$ value, the emulsification properties of these surfactants towards liquid paraffin were improved.

Foam was formed by air and other gases surrounded by liquid. With the increment of $R$ value, the foam properties of these surfactants were improved. Their initial foam height and stable foam height after 5 minutes increased as the $R$ value increased. The emulsification ability and foam ability greatly improved upon introduction of a hydrophobic rigid core into the polymeric surfactants.

\section{Rheological properties}

The shear stresses of $50 \mathrm{~g} \mathrm{~L}^{-1}$ of 950AA, 950BA, 950CA, and 950DA aqueous solutions as a function of shear rate at different temperatures $\left(30,45\right.$ or $\left.60{ }^{\circ} \mathrm{C}\right)$ are displayed in Fig. 3. The apparent viscosities of 950AA, 950BA, 950CA, and 950DA solutions could be determined by dividing shear stress by shear rate. It was clear that the solutions were dilatant fluids at the experimental concentration and temperature because the apparent viscosities of all four samples increased as the shear rate increased. ${ }^{16}$ Fig. 3 shows that the apparent viscosities of these surfactants decreased with an increment of temperature. When temperature was higher, the molecular aggregates moved faster, and the frequency of aggregate collision was greater. Another reason was that the hydration of the branched chain of the hydrophilic group decreased with an increase in temperature, and the interaction between the water molecules and the molecular aggregates reduced; this led to a decrease in viscosity. ${ }^{16}$

There was a break point that could be found in the apparent viscosity-shear rate curves in Fig. 3. The break point ranged from a shear rate of $150 \mathrm{~s}^{-1}$ to $220 \mathrm{~s}^{-1}$. This suggested that the shear stress cannot change the arrangement of the aggregates at lower shear rates. The lower viscosity was mainly because of water molecules moving through the network structure of aggregation. Relative movement between aggregates might occur when the shear rate reached where the break point was. The interaction of the branched chain of the hydrophilic group stretching out from the aggregates resulted in the increment of viscosity. Therefore, to break and rearrange the cross-linked structure, a higher shear rate is required. Although the change of apparent viscosities in Fig. 3 is similar among 950AA, 950BA, 950CA, and 950DA, the break point moves to a high shear rate with an increment of the $R$ value. The shear rate at the break point was $163 \mathrm{~s}^{-1}$ (950AA), $175 \mathrm{~s}^{-1}$ (950BA), $193 \mathrm{~s}^{-1}$ (950CA), and $212 \mathrm{~s}^{-1}$ (950DA). As the $R$ value increased, the break points moving to higher shear rates might be caused by the increment of aggregates interaction as abovementioned.

\section{Characterization of water suspension concentrates}

Pymetrozine water suspension concentrates were prepared by grinding using comb-like surfactants poly(RGMA-co-MAPEG) as the dispersant. The particle size, suspension rate, and viscosity of water suspension concentrate are shown in Table 3.

Compared with P-MAPEG (950AA), poly(RGMA-co-MAPEG) as a dispersant had better grinding and dispersion to $5 \%$ pymetrozine water suspension concentrates. Under the same grinding condition, the particle size of the water suspension concentrates using poly(RGMA-co-MAPEG) as the dispersant was smaller than that obtained using poly-MAPEG (950AA) as the dispersant. Particle size and particle size distribution of the suspension were determined by a laser diffraction particle size analyzer (Mastersizer 2000). The particle size dispersity of the suspension is displayed in Fig. S5. $\dagger$ The average particle size of the water suspension concentrates using 950BA or 950CA as the dispersant is smaller than $5 \mu \mathrm{m}$, which can meet international standard for the particle size of water suspension concentrates. It could be concluded that the introduction of the tricyclic diterpene skeleton into the surfactant resulted in a better grinding effect to the water suspension concentrates.

Compared with that of poly-MAPEG (950AA), the suspension rate of the water suspension concentrates was higher than $95 \%$ when poly(RGMA-co-MAPEG) was used as a dispersant. They can all meet the international standard of water

Table 2 Emulsification, foam properties, and HLB values of four surfactants

\begin{tabular}{|c|c|c|c|c|c|c|}
\hline \multirow[b]{2}{*}{ Products } & \multirow[b]{2}{*}{ Emulsifications (s) } & \multicolumn{2}{|c|}{ Foam properties (mm) } & \multirow[b]{2}{*}{ HLB values } & \multirow[b]{2}{*}{$\mathrm{CMC}\left(\mathrm{g} \mathrm{L}^{-1}\right)$} & \multirow[b]{2}{*}{$\gamma_{\mathrm{CMC}}\left(\mathrm{mN} \mathrm{m}^{-1}\right)$} \\
\hline & & $0 \mathrm{~min}$ & $5 \mathrm{~min}$ & & & \\
\hline 950BA & 525 & 100 & 61 & 18.6 & 0.004 & 57.8 \\
\hline 950CA & 570 & 103 & 71 & 18.2 & 0.011 & 56.6 \\
\hline 950DA & 640 & 125 & 88 & 17.6 & 0.023 & 51.9 \\
\hline
\end{tabular}



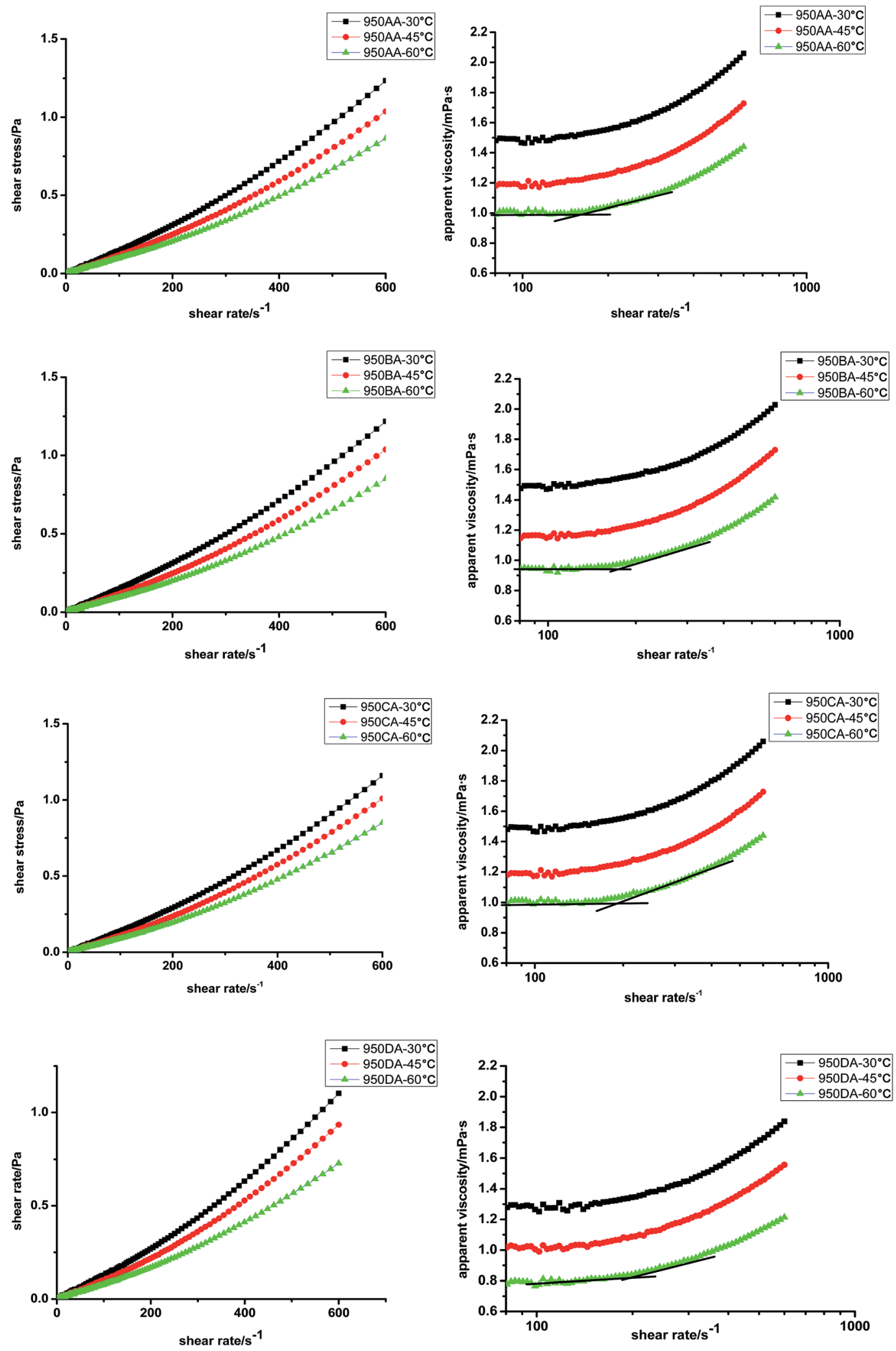

Fig. 3 Shear rate $\left(\mathrm{s}^{-1}\right)$ vs. shear stress $(\mathrm{Pa})$ and apparent viscosity (mPa s).

suspension concentrates. As the $R$ value increased, the suspension rate of the water suspension concentrates increased. It can be deduced that the introduction of the tricyclic diterpene skeleton into the surfactant can effectively improve the suspension rate of water suspension concentrates. After 15 days of cold storage, the suspension rate of water suspension concentrates was still greater than $95 \%$ while using 950BA or 950DA as the dispersant. 
Table 3 Particle size, suspension rate, and viscosity of pymetrozine water suspension concentrates on the 1st day and 15th day after cold storage

\begin{tabular}{|c|c|c|c|c|c|c|}
\hline & \multicolumn{2}{|c|}{ Average particle size $d_{43}(\mu \mathrm{m})$} & \multicolumn{2}{|c|}{$\underline{\text { Suspension rate }(\%)}$} & \multicolumn{2}{|c|}{ Viscosity (Pa s) } \\
\hline & 1st day & $\begin{array}{l}\text { 15th day after } \\
\text { cold storage }\end{array}$ & 1st day & $\begin{array}{l}\text { 15th day after } \\
\text { cold storage }\end{array}$ & 1st day & $\begin{array}{l}\text { 15th day after } \\
\text { cold storage }\end{array}$ \\
\hline 950AA & 13.34 & 37.67 & 39 & 79.8 & 146 & 209 \\
\hline 950BA & 1.27 & 0.65 & 95.7 & 95.5 & 165 & 216 \\
\hline 950CA & 1.21 & 1.31 & 95.9 & 89.2 & 247 & 260 \\
\hline
\end{tabular}

The introduction of the tricyclic diterpene skeleton into the surfactant has a significant effect on the viscosity of water suspension concentrates. As the $R$ value increased, the viscosity of the water suspension concentrates increased. However, the viscosity of the water suspension concentrates using 950DA as the dispersant was lower than that obtained using 950CA as the dispersant. This could be due to the sedimentation caused by the larger particle size. The viscosity of the water suspension concentrates increased after 15 days of cold storage. However, the thermal storage stability of the water suspension concentrates still needs to be improved.

In summary, 950BA possesses greater potential application in pymetrozine water suspension concentrates than others. Formulation of different types of dispersants with comb-like surfactants will be considered to further improve the performance of this type of water suspension concentrates. Pymetrozine water suspension concentrates will continue to be milled using 950BA as the dispersant and compounding it with other types of dispersants to improve the thermal storage stability.

\section{Conclusions}

A series of comb-like polymeric surfactants, poly(RGMA-coMAPEG), with a rigid core have been prepared from natural rosin, which is a type of natural forest resource. Poly(RGMA-co-MAPEG), with different molar ratios of RGMA to MAPEG $(R)$, was obtained by changing the feed ratio of RGMA to MAPEG. The test results of CMC values, emulsification, and foam ability of these polymeric surfactants indicated that introduction of a hydrophobic tricyclic rigid core into the polymeric surfactants can improve their surface activities. Dilatant behavior was observed in the $50 \mathrm{~g} \mathrm{~L}^{-1}$ comb-like polymeric surfactant solutions. There was a break point in the apparent viscosity-shear rate curve, and the break point moved to a higher shear rate as the proportion of hydrophobic tricyclic rigid core increased. The introduction of the rigid core of the tricyclic diterpene skeleton into the polymeric surfactants can effectively improve the suspension rate and stability of water suspension concentrates. Moreover, 950BA as a dispersant possesses greater potential application in pymetrozine water suspension concentrate than others because pymetrozine water suspension concentrates exhibit smaller particle size, higher suspension rate, lower viscosity, and better storage stability. We will continue to investigate pymetrozine water suspension concentrates using 950BA as a dispersant and compounding it with other types of dispersants to improve the thermal storage stability. This study may open a new application area of natural rosin.

\section{Conflicts of interest}

There are no conflicts of interest to declare.

\section{Acknowledgements}

This research was financially supported by the grants received from the National Natural Science Foundation of China (31470596 and 31622017) and Fundamental Research Foundation of the Central Commonwealth Institute of the Chinese Academy of Forestry (CAFYBB2012038).

\section{References}

1 P. Castelani, Oil Dispersion Formulations: Stability Assessment and Field Trials, 2016.

2 S. S. Baseeth, T. R. Tabuena-Salyers and B. R. Sebree, Dispersants having biobased compounds, US pat. US9517442, 2016.

3 I. Moreno-Garrido, M. Hampel, L. Lubián and J. Blasco, Fresenius. J. Anal. Chem., 2001, 371, 474-478.

4 P. Raffa, D. A. Wever, F. Picchioni and A. A. Broekhuis, Chem. Rev., 2015, 115, 8504.

5 K. N. Jayachandran, S. Maiti and P. R. Chatterji, Polymer, 2001, 42, 6113-6118.

6 R. Wang, H. Mei, W. Ren and Y. Zhang, RSC Adv., 2016, 6, 25-51. 7 V. Tomanová, I. Sroková, A. Ebringerová and V. Sasinková, Polym. Eng. Sci., 2011, 51, 1476-1483.

8 N. H. Sahu, P. M. Mandaogade, A. M. Deshmukh, V. S. Meghre and A. K. Dorle, J. Bioact. Compat. Polym., 1999, 14, 344-360.

9 N. S. Ahmed, J. Dispersion Sci. Technol., 2009, 30, 1100-1110. 10 X. Huang, H. Liu, S. Shang, X. Rao and J. Song, China Surfactant Deterg. Cosmet., 2015, 63, 9062-9068.

11 Z. Liu and G. Biresaw, J. Agric. Food Chem., 2011, 59, 1909-1917. 12 S. Li, S. Yan, J. Yu and B. Yu, J. Appl. Polym. Sci., 2011, 122, 2843-2848.

13 W. Zhang, Z. Du, C. H. Chang and G. Wang, J. Colloid Interface Sci., 2009, 337, 563.

$14 \mathrm{X}$. Huang, H. Liu, S. Shang, Z. Cai and J. Song, RSC Adv., 2016, 6, 68592.

15 S. S. Hou and P. L. Kuo, Polymer, 2001, 42, 2387-2394.

16 K. Holmberg, J. Bo, B. Kronberg and B. Lindman, SurfactantPolymer Systems, John Wiley \& Sons, Ltd, 2003, ch. 13. 